\title{
Instabilities of high-order beam modes driven by space-charge coupling resonances
}

\author{
A. V. Fedotov, J. A. Holmes, and R. L. Gluckstern \\ CA Department, Brookhaven National Laboratory, Upton, New York 11973, \\ SNS Project, Oak Ridge National Laboratory, Oak Ridge, Tennessee 37831, \\ and Physics Department, University of Maryland, College Park, Maryland 20742
}

(Received 24 April 2001; published 10 August 2001)

\begin{abstract}
Space charge presents a fundamental limitation to high intensity circular accelerators. Its effects are especially important in the latest designs for high-intensity proton rings, which require beam losses much smaller than presently achieved in existing facilities. It is therefore necessary to understand the major space-charge effects which could lead to emittance growth and associated beam loss. In this paper, we explore the excitation of high-order collective beam modes and associated instabilities driven by space-charge coupling resonances. Such studies help us to understand energy exchange and emittance growth driven by space-charge coupling. They also have direct application to the choice of a good working point in a high-intensity machine. The studies are performed using an earlier version of the Spallation Neutron Source lattice, which was used as a generic example of a circular machine. In this way, we explore the nature of the observed space-charge coupling effect and its applicability to highintensity rings in general.
\end{abstract}

DOI: 10.1103/PhysRevSTAB.4.084202

PACS numbers: 29.27.Bd

\section{INTRODUCTION}

Our studies were stimulated by the fourth order resonance structure observed in simulations with a KV beam for a previous version of the Spallation Neutron Source (SNS) lattice [1]. The observed structure was attributed to a resonance with harmonic $N=23$. However, the SNS lattice has superperiodicity equal to 4 , and, thus, the appearance of harmonic 23 in the absence of magnet errors is not expected. We have therefore undertaken a detailed study of this issue in an attempt to explain the nature of the observed resonance [1].

Starting with a simple frequency analysis (see the Appendix) of the simulation in question, we confirm that harmonic 23 is not present. Further simulations demonstrate that the observed structure is not associated with a specific tune but instead is driven by nonlinear space-charge $x-y$ coupling. This conclusion is followed by a systematic study of the coupling resonance for different tunes, which results in the observation of instabilities for various high-order beam modes. These instabilities and the corresponding resonances were recently described by Hofmann analytically [2] and confirmed numerically [3] with reference to a high-intensity linear accelerator in a very different space-charge regime from the one of interest in rings. Understanding the impact of these instabilities on emittance growth is very important for high-intensity circular machines. It becomes of special interest since the region near the $\nu_{x}=\nu_{y}$ resonance line is the largest region free of low-order magnet imperfection resonances. In this paper, we present a detailed numerical investigation of the excitation of these high-order collective modes for beam parameters of an earlier version of the SNS accumulator ring [4]. We note that a special feature of the SNS is multiturn injection with final full intensity reached just before extraction, without subsequent storage of the beam. As a result, the studies presented here, which are based on full-intensity beams, are not directly applicable to the SNS, but they may be relevant to other accelerators with long-time beam storage.

\section{EXCITATION OF HIGH-ORDER MODES IN THE PRESENCE OF SPACE-CHARGE COUPLING}

Most of our studies were performed using the ORBIT code [5]. First, we reproduced the fourth order beam structure for a $\left(\nu_{x}, \nu_{y}\right)=(5.82,5.79)$ working point. Exploration of this structure shows that it is not related to any resonance with the lattice harmonic. Instead, we observed excitation of the fourth order collective beam mode $\Omega_{4}$ (see the Appendix). This mode is excited only in the presence of $x-y$ coupling. The bandwidth of this coupling resonance and its effect on excitation of the $\Omega_{4}$ mode are thoroughly investigated and summarized in Fig. 1, which plots the changes in saturated horizontal and vertical rms emittances as a function of $\nu_{y}$ for $\nu_{x}=5.82$. The initial rms emittances are both $30 \pi \mathrm{mm} \mathrm{mrad}$, and the figure shows an overall width of about $\delta \nu_{y}=0.1$. Such a small bandwidth is due to the very weak (few percent) tune depression which is typical in high-intensity rings. Figure 1 also shows energy exchange for slightly nonequipartitioned initial distributions. We note that simulations were performed for the unbunched KV beam with a small energy spread $\Delta p / p \approx 0.7 \%$, which accounts for some asymmetry of the resonant exchange in Fig. 1.

We then consider various combinations of tunes, and observe excitation of high-order collective modes when the 


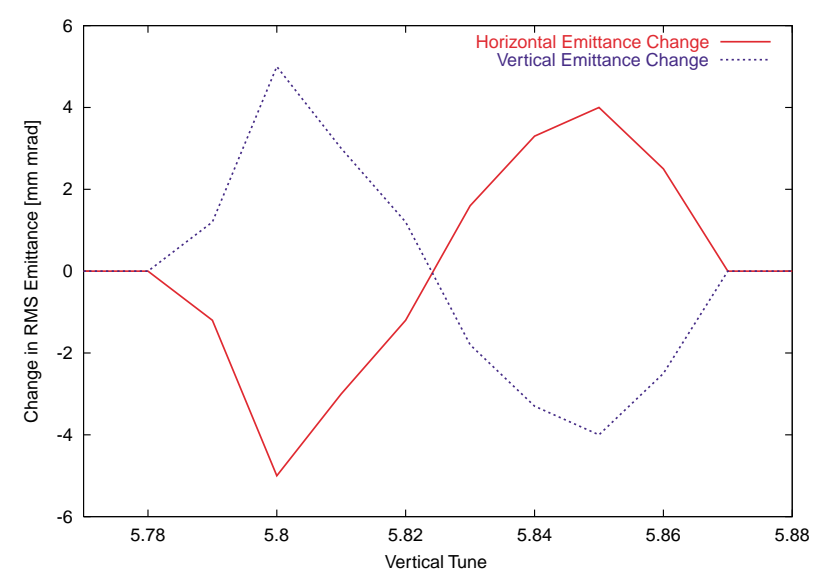

FIG. 1. (Color) The change of the horizontal and vertical rms emittances as a function of zero-current tune $\nu_{y}$ for fixed $\nu_{x}=5.82$.

coupling is strong. In fact, both the third and fourth order modes are observed, which agrees with the theoretical description for a KV beam [2]. In all cases, we perform a frequency analysis which demonstrates that these resonances do not occur at the betatron lattice harmonics. Rather, we observe an excitation of the corresponding $m$ th order coherent modes, as confirmed by the frequency content.

\section{Various combinations of working points}

We now demonstrate the excitation of high-order modes for various working points. Following a discussion with Hofmann [6], the excitation of third or fourth order modes is not related to the tunes, but is rather determined by the asymmetries contained in the numerically generated initial distribution and by the slightly different beam parameters resulting from the rms matching procedure. We should also note that, for our beam parameters, the unstable regions [2] of the fourth order modes are more pronounced than those of the third order modes. Here we do not discuss the classification of the modes which become unstable and refer the interested reader to Ref. [2].

$$
\text { 1. }\left(\nu_{x}, \nu_{y}\right)=(5.82,5.79)
$$

In this example, we demonstrate the resonance effect associated with an excitation of the coherent beam mode by providing a set of figures: time evolution of rms emittances and beam moments, frequency analysis of beam moments and phase-space plots. For other examples only selected figures from this set are shown.

For this working point, we observe a fourth order resonance structure in the vertical plane around 400 turns. The working point $\left(\nu_{x}, \nu_{y}\right)=(5.82,5.79)$ results in depressed tunes around $\left(\bar{\nu}_{x}, \bar{\nu}_{y}\right)=(5.75,5.72)$. With rms emittances $\tilde{\boldsymbol{\epsilon}}_{x} \approx \tilde{\boldsymbol{\epsilon}}_{y}$, for $\nu_{y}<\nu_{x}$, the vertical beam size $b$ is larger than the horizontal beam size $a$, and the beam temperature $T_{y}=\epsilon_{y} \nu_{y}<T_{x}=\epsilon_{x} \nu_{x}$. For $\nu_{y}=5.79$, analytic the- ory [2] predicts a frequency for the antisymmetric fourth order beam mode of $\Omega_{4}=22.8$. Frequency analysis of the time evolution of the fourth order moment $\left\langle y^{4}\right\rangle$ in the simulation also shows a peak at this frequency, as can be seen in Fig. 2. The time evolution of rms emittances, second order moments, and fourth order moments are shown in Figs. 3-5, respectively. These clearly show the energy exchange facilitated by the strong coupling, and the

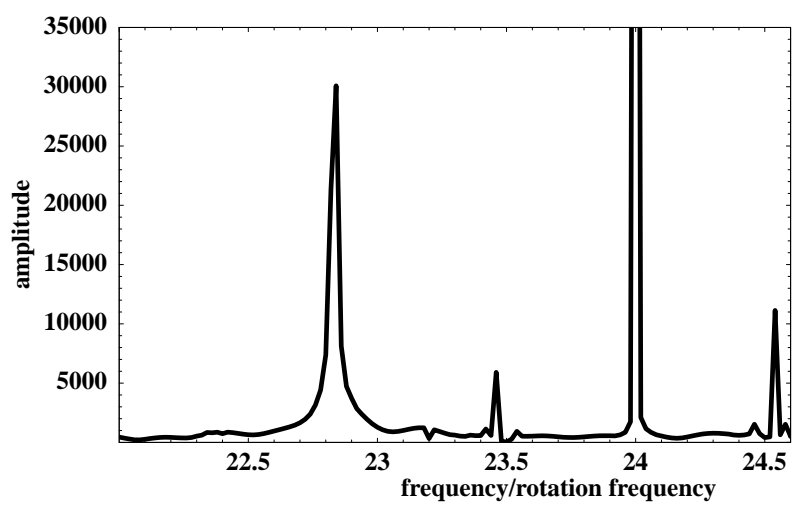

FIG. 2. Spectrum of the fourth order moments $\left\langle y^{4}\right\rangle$ at 375 turns for $\left(\nu_{x}, \nu_{y}\right)=(5.82,5.79)$.

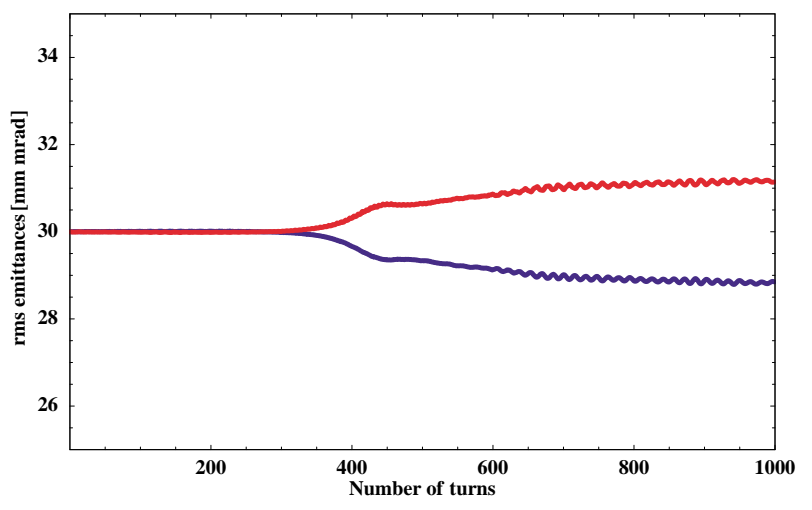

FIG. 3. (Color) Time evolution of rms emittance for $\left(\nu_{x}, \nu_{y}\right)=$ $(5.82,5.79)$. $X$ emittance is shown in blue and $Y$ emittance in red.

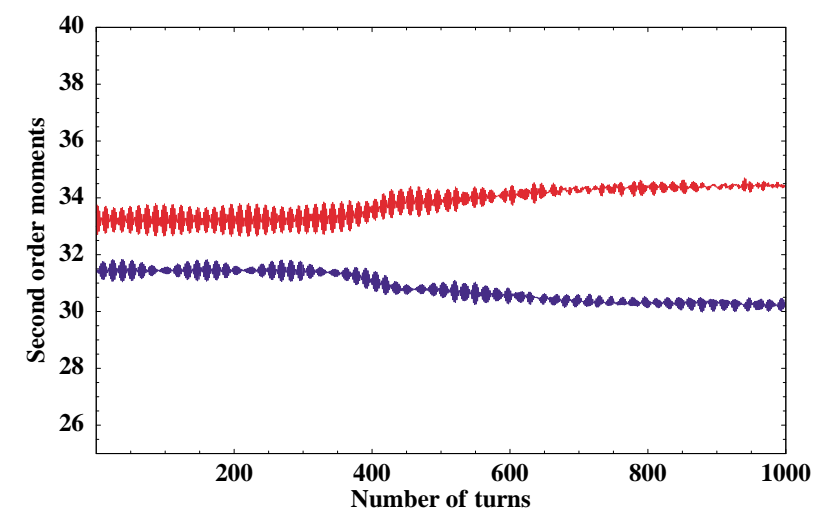

FIG. 4. (Color) Time evolution of $\left\langle x^{2}\right\rangle$ (blue) and $\left\langle y^{2}\right\rangle$ (red) for $\left(\nu_{x}, \nu_{y}\right)=(5.82,5.79)$ in normalized units. 
excitation of the fourth order mode is also clear in the $\left\langle y^{4}\right\rangle$ moments. Figure 6 shows the fourth order mode already developed by 375 turns. At a later stage, individual particles resonate with this collective mode, as shown in Fig. 7, at 500 turns.

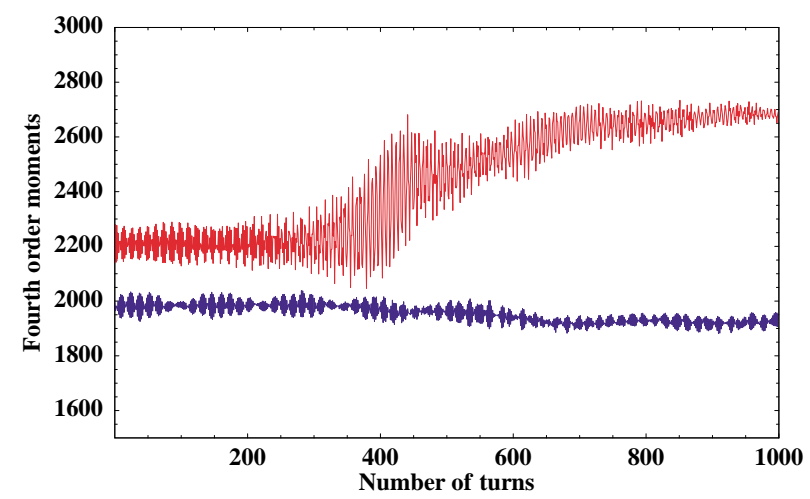

FIG. 5. (Color) Time evolution of $\left\langle x^{4}\right\rangle$ (blue) and $\left\langle y^{4}\right\rangle$ (red) for $\left(\nu_{x}, \nu_{y}\right)=(5.82,5.79)$ in normalized units.

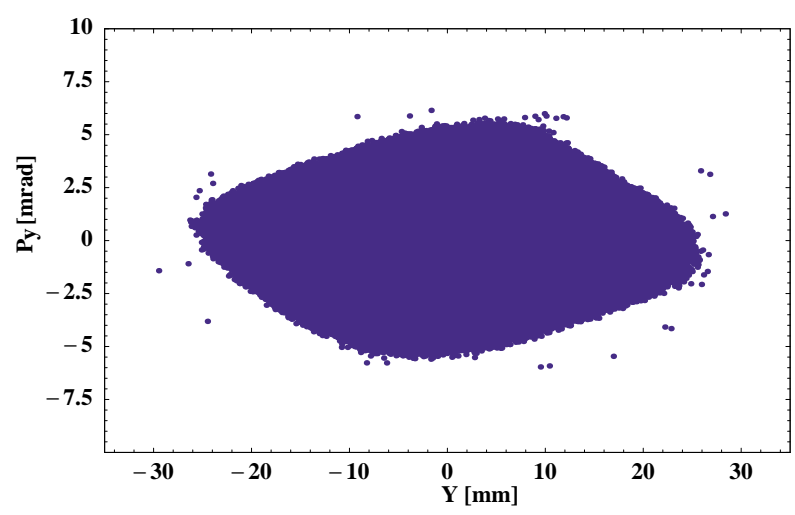

FIG. 6. (Color) $Y-P_{y}$ phase-space plot at 375 turns for $\left(\nu_{x}, \nu_{y}\right)=(5.82,5.79)$.

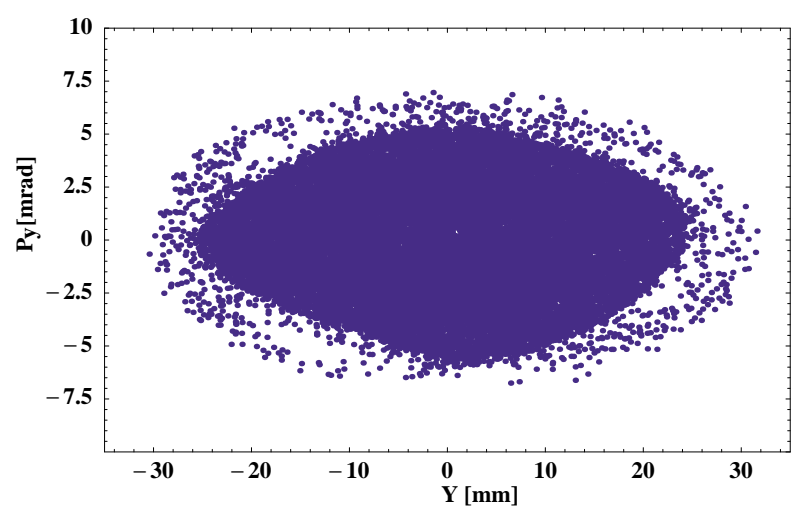

FIG. 7. (Color) $Y-P_{y}$ phase-space plot at 500 turns for $\left(\nu_{x}, \nu_{y}\right)=(5.82,5.79)$.
2. $\left(\nu_{x}, \nu_{y}\right)=(5.57,5.54)$

In this case, we observe a third order resonance structure in the vertical plane around 400 turns. For $\nu_{y}=5.54$, analytic theory predicts $\Omega_{3}=16.4$. Frequency analysis of the time evolution of $\left\langle y^{3}\right\rangle$ also shows a peak at this frequency, as can be seen in Fig. 8. The time evolution of rms

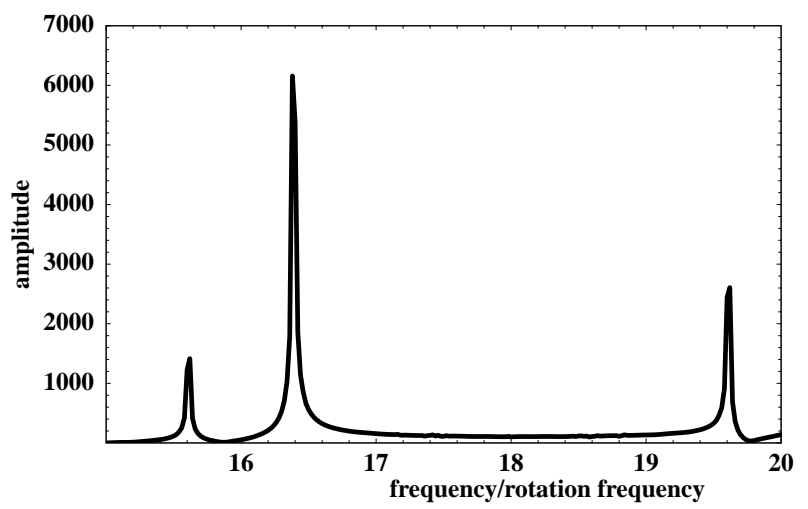

FIG. 8. Spectrum of $\left\langle y^{3}\right\rangle$ at 375 turns for $\left(\nu_{x}, \nu_{y}\right)=$ $(5.57,5.54)$.

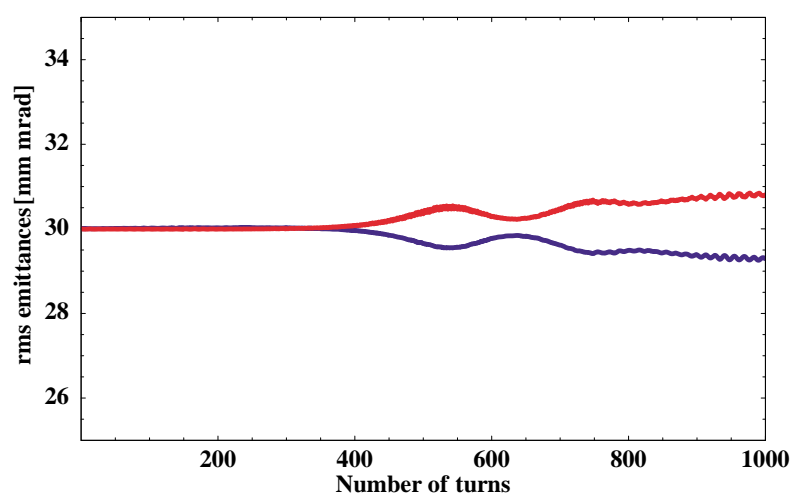

FIG. 9. (Color) Time evolution of rms emittance for $\left(\nu_{x}, \nu_{y}\right)=$ (5.57,5.54). $X$ emittance is shown in blue and $Y$ emittance is shown in red.

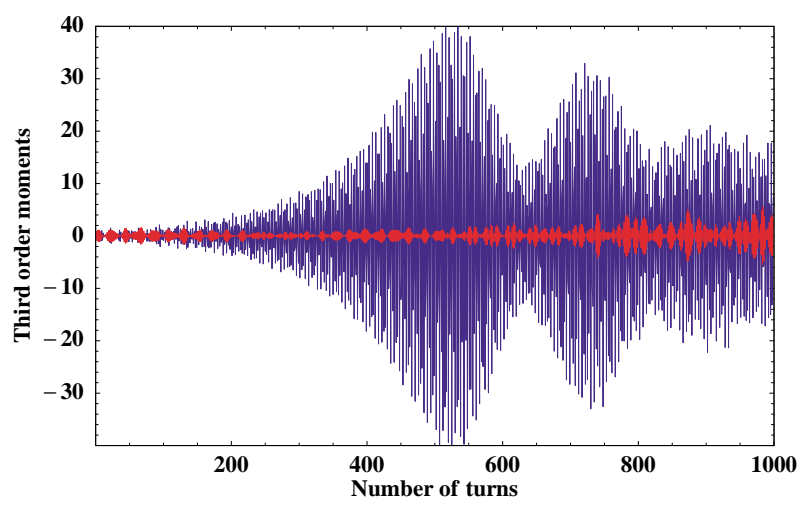

FIG. 10. (Color) Time evolution of $\left\langle x^{3}\right\rangle$ (red) and $\left\langle y^{3}\right\rangle$ (blue) for $\left(\nu_{x}, \nu_{y}\right)=(5.57,5.54)$ in normalized units. 
emittances and third order moments are shown in Figs. 9 and 10, respectively. Again, the energy exchange and excitation of the third order coherent mode via coupling resonance are clear. The corresponding $y-y^{\prime}$ phase-space plot reveals the third order structure.

$$
\text { 3. }\left(\nu_{x}, \nu_{y}\right)=(5.82,5.86)
$$

Here, we observe a third order resonance structure in the horizontal plane. With equal initial emittances in the two planes and $\nu_{x}<\nu_{y}$, we have $T_{y}=\epsilon_{y} \nu_{y}>T_{x}=\epsilon_{x} \nu_{x}$. For $\nu_{x}=5.82$, analytic theory predicts $\Omega_{3}=17.3$. Frequency analysis of the time evolution of $\left\langle x^{3}\right\rangle$ in simulations also shows a peak at this frequency, as can be seen in Fig. 11. The time evolution of the rms emittances and third order moments are shown in Figs. 12 and 13, respectively. The emittance evolution demonstrates the expected energy exchange, and the third order moments dramatically illustrate the instability.

This case of an odd order mode excitation is similar to the one presented by Hofmann [3]. The vertical beam size is still larger than the horizontal beam size but, as stated

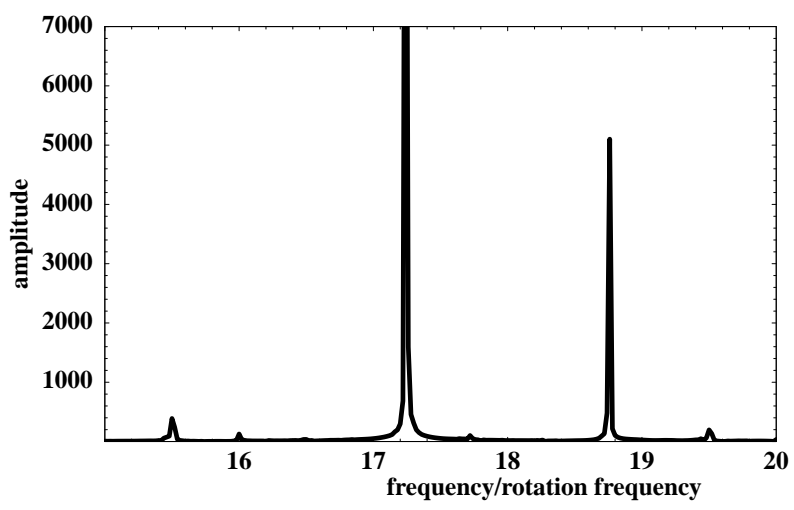

FIG. 11. Spectrum of $\left\langle x^{3}\right\rangle$ at 375 turns for $\left(\nu_{x}, \nu_{y}\right)=$ $(5.82,5.86)$.

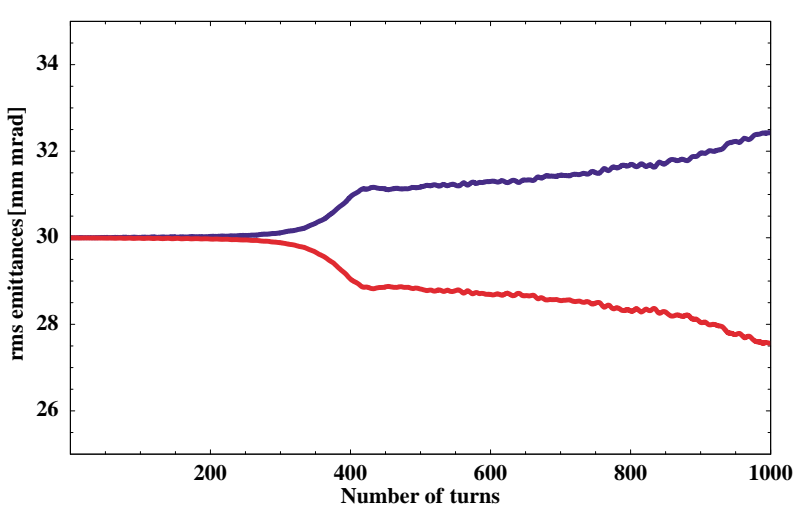

FIG. 12. (Color) Time evolution of rms emittance for $\left(\nu_{x}, \nu_{y}\right)=$ $(5.82,5.86)$. $X$ emittance is shown blue and $Y$ emittance is shown in red.

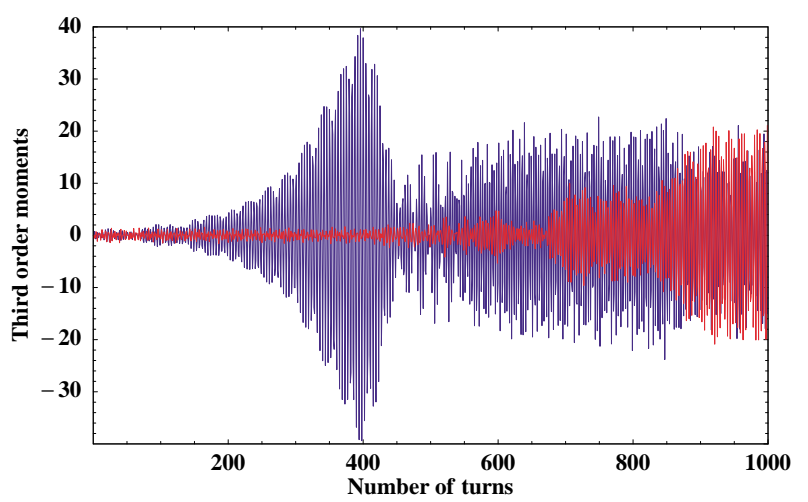

FIG. 13. (Color) Time evolution of $\left\langle x^{3}\right\rangle$ (blue) and $\left\langle y^{3}\right\rangle$ (red) for $\left(\nu_{x}, \nu_{y}\right)=(5.82,5.86)$ in normalized units.

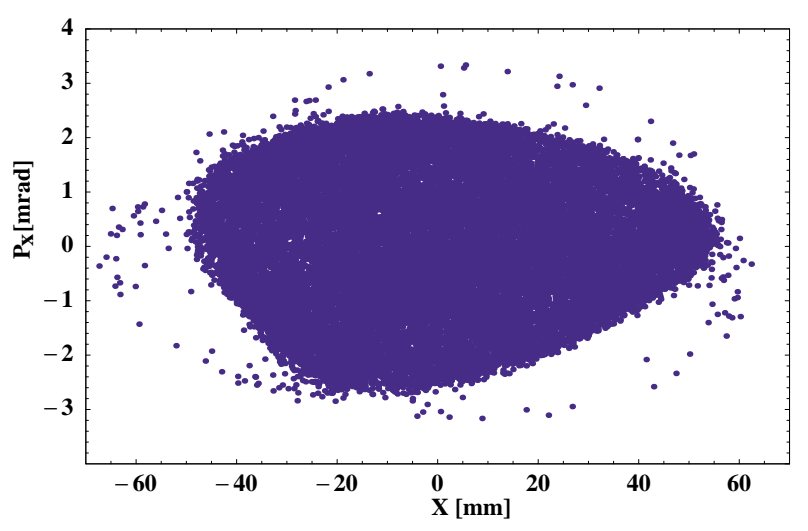

FIG. 14. (Color) $X-P_{x}$ phase-space plot at 375 turns for $\left(\nu_{x}, \nu_{y}\right)=(5.82,5.86)$.

above, $T_{x}<T_{y}$, which leads to the deformation of beam envelopes and excitation of the third order odd mode. A corresponding phase-space plot of $x-x^{\prime}$ from the present simulation at 375 turns is shown in Fig. 14.

$$
\text { 4. }\left(\nu_{x}, \nu_{y}\right)=(5.74,5.71)
$$

For this working point, we first observe a fourth order resonance structure in the vertical plane after about 300 turns and subsequent development of a third order structure after 600 turns. With $\nu_{y}=5.71$, analytic theory predicts $\Omega_{3}=22.5$. Frequency analysis of the time evolution of $\left\langle y^{4}\right\rangle$ around 375 turns in the simulation shows a peak exactly at this frequency, as can be seen in Fig. 15. Similarly, for $\nu_{y}=5.71$, the frequency of the third order coherent mode is $\Omega_{3}=16.9$. Frequency analysis of the time evolution of $\left\langle y^{3}\right\rangle$ at 1000 turns in simulations shows a peak at this frequency, as can be seen in Fig. 16. Corresponding phase-space structures can be seen in Figs. 17 and 18 at 375 and 1000 turns, respectively. The time evolution of the rms 


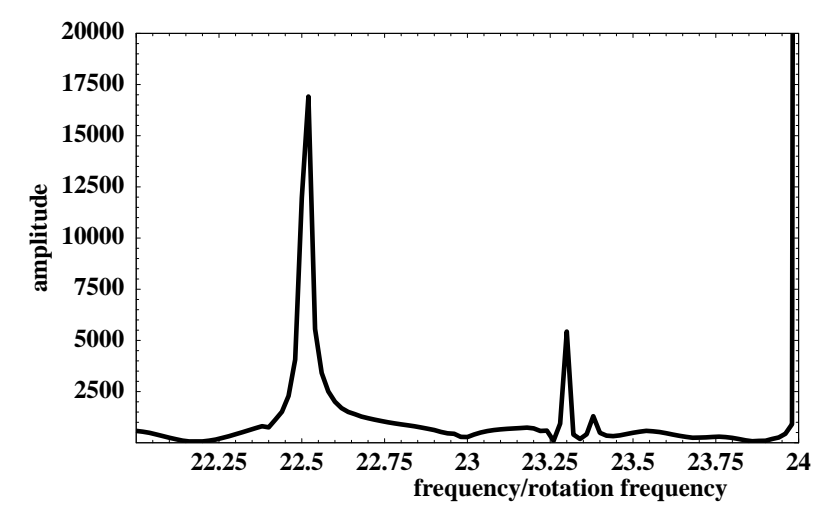

FIG. 15. Spectrum of $\left\langle y^{4}\right\rangle$ at 375 turns for $\left(\nu_{x}, \nu_{y}\right)=$ $(5.74,5.71)$.

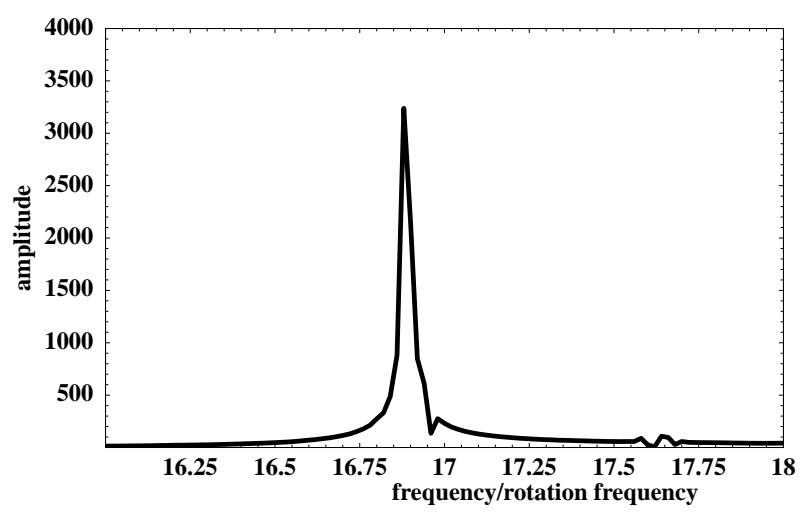

FIG. 16. Spectrum of $\left\langle y^{3}\right\rangle$ at 1000 turns for $\left(\nu_{x}, \nu_{y}\right)=$ $(5.74,5.71)$.

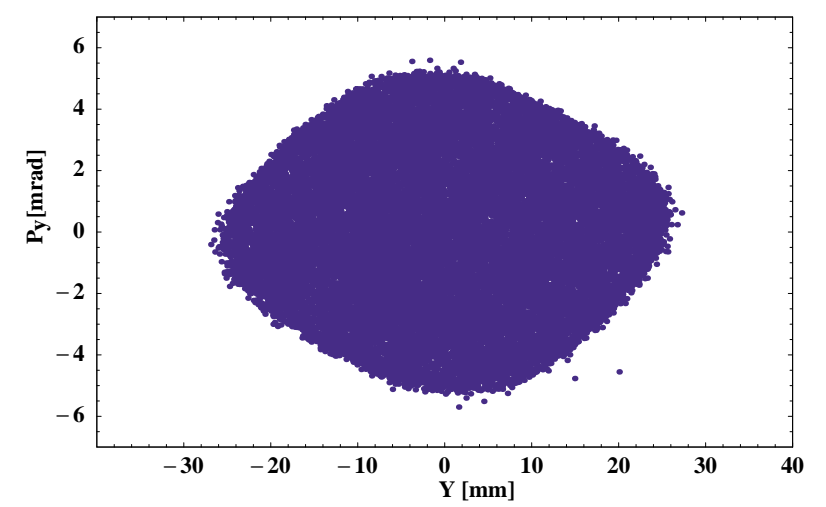

FIG. 17. (Color) $Y-P_{y}$ phase-space plot at 375 turns for $\left(\nu_{x}, \nu_{y}\right)=(5.74,5.71)$.

emittances, third order moments, and fourth order moments show that the excitation of the fourth order moments precedes that of the third order moments. This case demonstrates that both even and odd modes can be excited.

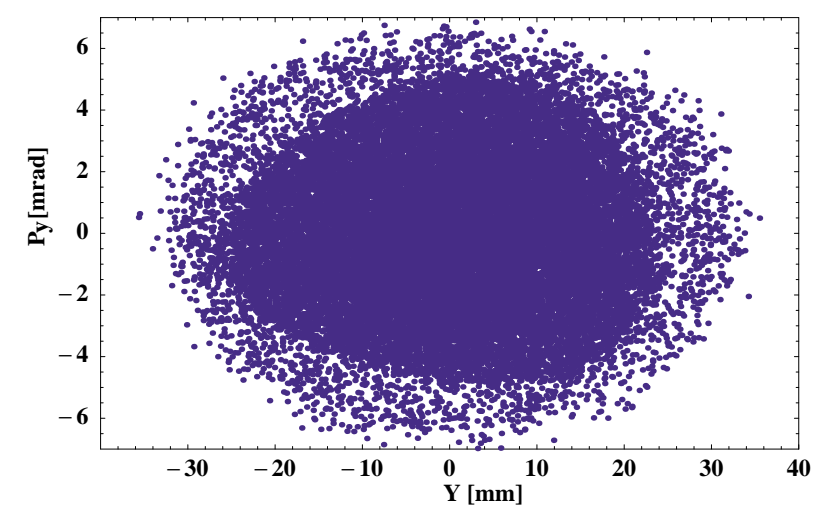

FIG. 18. (Color) $Y-P_{y}$ phase-space plot at 1000 turns for $\left(\nu_{x}, \nu_{y}\right)=(5.74,5.71)$.

\section{EXCITATION OF ODD ORDER MODES}

Despite the fact that the KV distribution has global symmetry, one may excite both odd and even order modes when this distribution is perturbed. For a numerically generated KV beam, it is not surprising that small initial asymmetries can lead to an excitation of odd order modes and become amplified in the instability process, as described in [2]. Thus, one would expect that odd order modes can be excited when the parameters are inside the instability region, and some asymmetry is present in the initial distribution. To confirm this expectation, we considered a case in which an odd order mode became excited. We recalculated this case with one change only: we forced the initial distribution to be exactly symmetric in the distribution generator, with all other parameters kept fixed. The resulting excitation contained only even modes, and the initial symmetry was precisely preserved. Because the growth rate of these instabilities increases with intensity, it is possible to observe the excitation of high-order modes on much shorter time scales by increasing the beam intensity. This was done in later simulations.

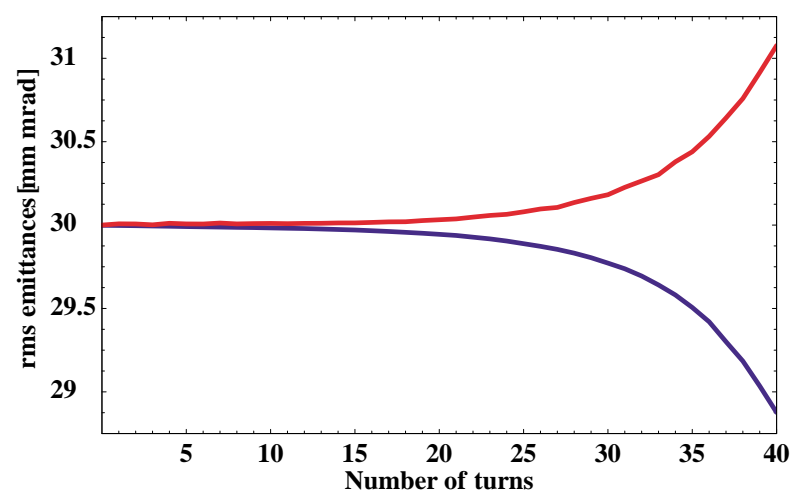

FIG. 19. (Color) ACCSIM run for the distribution with initial asymmetry. Time evolution of rms emittances for working point with zero-current tunes $\left(\nu_{x}, \nu_{y}\right)=(5.82,5.75)$. Emittance in $x$ is shown in blue and emittance in $y$ is shown in red. 
During the course of these studies an additional concern surfaced [7] that excitation of odd order modes could be a feature of the code which we are using. Therefore, similar cases were run with the ACCSIM code [8]. In these runs, beam intensity and corresponding space-charge tune shift were increased by approximately a factor of 2 . Also, the ratio of full-current tunes was chosen in accordance with Fig. 1 to have a significant emittance exchange. As a result, emittance exchange was observed on much faster time scale. Figures 19 and 20, obtained from an ACCSIM calculation, show an excitation of the third order (odd) beam mode when there is an initial asymmetry in the numerically generated KV distribution. Figure 19 shows an exchange of rms emittances between the two transverse planes, and Fig. 20 shows development of an odd order mode. In fact, this odd mode structure is strikingly similar to the odd mode observed in [3]. When the initial distribution is forced to be symmetric by the distribution generator, for the same case, we observe excitation of the even mode instead, as demonstrated in Fig. 21.

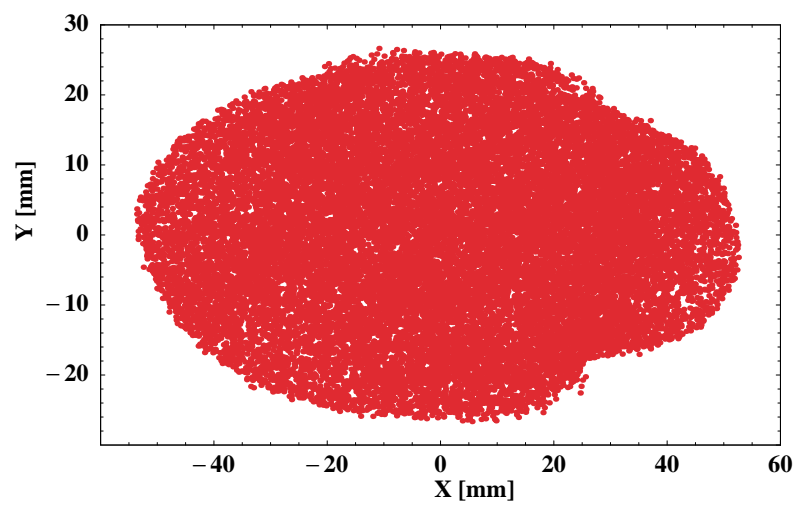

FIG. 20. (Color) ACCSIM run for the distribution with initial asymmetry. $X-Y$ projection after 40 turns (working point with zero-current tunes 5.82,5.75).

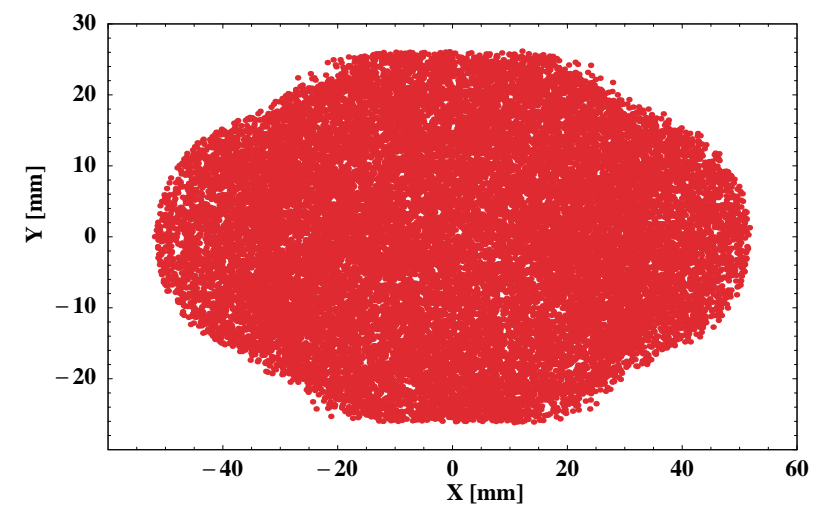

FIG. 21. (Color) ACCSIM run for initially symmetric distribution. $X-Y$ projection after 40 turns.

\section{MAJOR FEATURES OF COUPLING RESONANCE EXCITATION}

This section provides additional arguments that support our explanation that the observed structures and associated emittance growth appear to be due to an excitation of high-order coherent beam modes via the space-charge coupling resonance, with a subsequent resonant interaction of individual particles and coherent beam modes. For this reason, we refer to the latter stage of this process (after the coherent mode is developed) as an intrinsic resonance. The space-charge coupling effect discussed here for a $\mathrm{KV}$ beam demonstrates the difference between the single-particle approach and the coherent mode approach adopted by Hofmann [2]. Based on the single-particle approach (Montague resonance [9]), even a numerical KV beam does not have sufficient nonlinear strength to provide coupling. However, the collective mode approach $[2,10]$ clearly demonstrates that small initial fluctuations in $\mathrm{KV}$ distribution can grow exponentially, thus providing a mechanism for space-charge coupling. Some important features are summarized below.

(i) The rms mismatch of the beam corresponds to an excitation of second order (envelope) modes which results in a parametric 1:2 (the frequency of individual particles is half the envelope frequency) resonance. This becomes the dominant effect making the observation of intrinsic resonances with high-order collective modes possible only in the absence of significant rms mismatch. This statement was confirmed in our simulations: the results shown here were obtained from the evolution of carefully matched initial distributions. The use of even mildly mismatched initializations led to strong and dominant intrinsic resonance with the second order mode. This is explained by the fact that, for very weak tune depression (around 2\%), the growth rate of instabilities, which drive high-order collective beam modes, is very slow.

(ii) Another feature of this coupling resonance is the time evolution of the process. Excitation of a high-order coherent beam mode and subsequent intrinsic resonance requires several hundred turns for low space charge (typical tune depression in high-intensity rings 1\%-3\%). On the contrary, resonance of individual particles with the lattice harmonics would occur much faster.

(iii) For a KV beam, the distribution employed in these studies, there would be significant difference between an intrinsic resonance with slowly developed high-order collective mode and a resonance with the lattice harmonic. Because all particles in a KV beam have similar frequencies, resonance with a lattice harmonic would be of a coherent nature, with all particles responding in a coherent way. In the intrinsic resonance, only a small portion of the beam enters a beam halo due to the nonlinear detuning. This agrees with the results of present simulations, as well as with the well established picture of parametric halo formation in high-intensity linacs. 


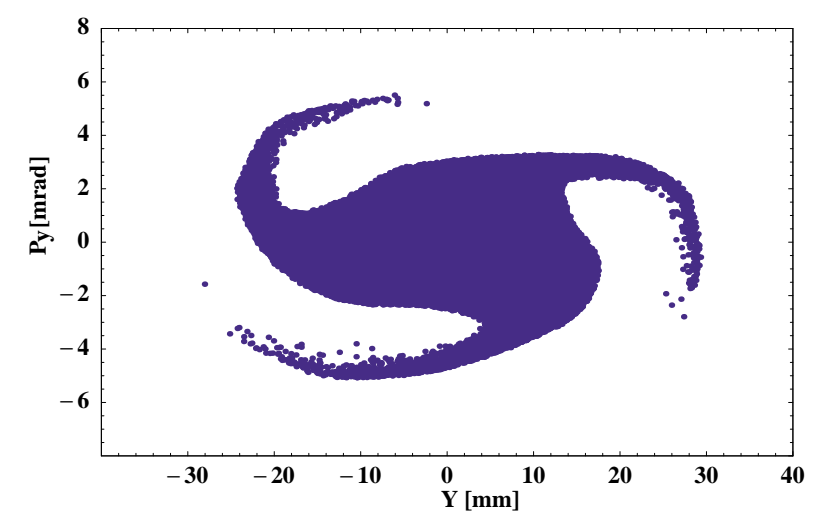

FIG. 22. (Color) Third order lattice resonance for KV beam only after 10 turns.

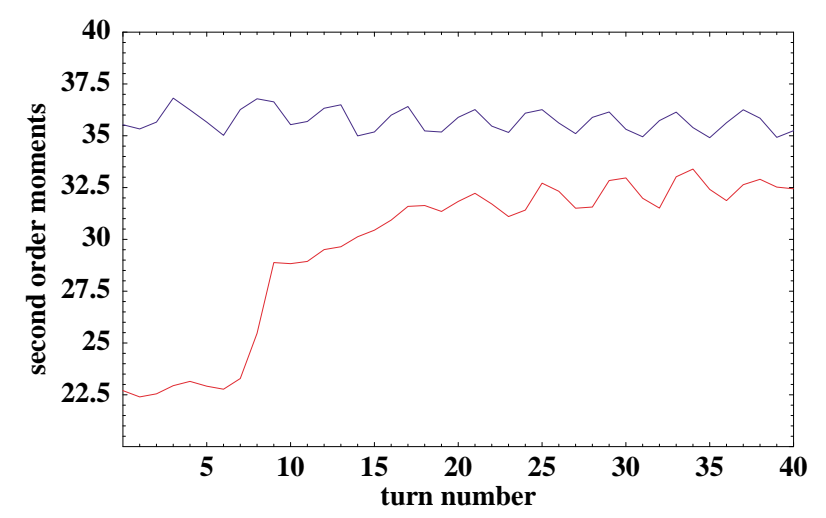

FIG. 23. (Color) Time evolution of second order beam moments (envelopes) with very fast third order lattice resonance (in normalized units). Horizontal beam envelope (blue) and vertical beam envelope (red).

For completeness, we now demonstrate the response of a KV beam to a lattice resonance. For this purpose, we increased the beam intensity and changed the beam aspect ratio in such a way that the depressed vertical tune becomes close to the lattice harmonic $n=16$. We now expect a very fast coherent response of the KV beam. This is clearly demonstrated in Figs. 22 and 23. Figure 22 shows the vertical phase space after 10 turns. One can see the coherent response of a $\mathrm{KV}$ beam to a lattice resonance. The corresponding fast blowup of the beam envelope is shown in Fig. 23. We again alter the beam intensity so that the depressed tune in $y$ becomes close to the fourth order resonance with lattice harmonic $n=20$, and the depressed tune in $x$ is near the sixth order resonance with $n=32$. The observed resonances again clearly demonstrate the fast and coherent response of a KV beam. Such resonances are well-known lattice resonances and should not be mixed with the space-charge coupling resonant excitation discussed in this paper.

\section{APPLICATIONS}

\section{A. SNS accumulator ring}

The space-charge coupling effect, described in this paper, is based on the studies of full intensity beams. In the SNS, accumulation is done via multiturn injection with the final intensity reached only at the end of the injection process. The beam is then extracted without a long storage time. The growth rates of the instabilities considered here are very slow, even for full-current beams (which correspond to $2 \%$ tune depression) compared to the highintensity linacs. In addition, the multiturn injection painting may easily wash out the resonant excitation, described in this paper, due to phase mixing and injection mismatch. As a result, the instability process observed here is not expected for the SNS accumulator ring beam parameters and the types of working points discussed in this paper.

\section{B. High-intensity storage rings}

In this section, we discuss whether the instability process studied in this paper should be expected in highintensity rings with long time storage of full intensity beams. We note that the studies presented here are based on the KV beam. The KV distribution is an excellent distribution to use for analytic calculation, but it is not very realistic. We therefore performed similar simulations with the Waterbag and Gaussian distributions, for which we did not observe instabilities of high-order beam modes, which are the subject of present studies. This is not surprising since realistic distributions have frequency spread and, thus, effective Landau damping. A more detailed explanation could be given based on the recent studies [11], which indicate that one type of unstable mode observed for a KV beam gets damped for non-KV distributions. The other type of modes, which can lead to similar instabilities in realistic non-KV beams, has different instability regions and requires much stronger tune depression to become unstable. The situation also changes when the emittances in the two planes differ significantly. For completeness, we note that simulations presented in this paper were done for an unbunched $\mathrm{KV}$ beam with a small energy spread of $\Delta p / p \approx 0.7 \%$. This slightly modified the tune shifts of individual particles and added the tune spread. In this paper, we do not study the effect of significant energy spread or synchrotron motion which could significantly alter the excitation of high-order coherent modes. For the SNS beam, some discussion of energy spread and its combined effect with space charge can be found in $[12,13]$. To summarize, for beam parameters discussed in this paper, the unstable high-order collective modes are expected mainly for the KV distribution rather than for realistic beams. However, for high-intensity circular accelerators with significant tune depression and/or different transverse emittances, the excitation of high-order collective beam modes and 
associated emittance growth could be a potential source of beam halo, and, thus, this effect should be carefully explored.

\section{SUMMARY}

This paper explains the nature of the previously observed resonant structure for the former SNS working point with close transverse tunes [1]. It is found that various high-order collective beam modes can be excited as long as the instability condition is satisfied in the vicinity of the space-charge coupling resonance. The excitation of such modes and associated resonances was thoroughly investigated and presented in this paper. These studies significantly contribute to our understanding of the space-charge coupling resonances and have direct application to the choice of a working point in future high intensity circular accelerators.

\section{ACKNOWLEDGMENTS}

We are indebted to I. Hofmann for extensive comments and careful review of this manuscript. We are also very grateful to J. Wei and Y. Papaphilippou for many useful discussions during these studies. In addition, we thank D. Jeon for numerous comments and questions which led to this work.

\section{APPENDIX: FREQUENCY ANALYSIS}

The observed fourth order structure was first attributed to a nonstructure resonance with an integer lattice harmonic excited by the space charge [1]. However, the appearance of such harmonics for the ideal lattice with four superperiods in the absence of magnet errors is problematic. We thus derive expressions for the sidebands of the lattice harmonics due to space charge, starting with the analysis of the matched beam in the absence of lattice errors.

\section{A. Vertical motion}

We assume a $\mathrm{KV}$ beam with transverse dimensions $\sigma_{x}, \sigma_{y}$. The corresponding single-particle equation of motion is

$$
y^{\prime \prime}+K_{y}(s) y=\frac{2 I}{\sigma_{y}\left(\sigma_{x}+\sigma_{y}\right)} y,
$$

where $I$ is the space-charge parameter. Using the transformation $y=\sqrt{\beta_{y}} v, d \phi=d s /\left(\beta_{y} \nu_{y}\right)$, the equation of motion becomes

$$
\ddot{v}+\nu_{y}^{2} v=\frac{2 I}{\sigma_{y}\left(\sigma_{x}+\sigma_{y}\right)} \beta_{y}^{2} \nu_{y}^{2} v,
$$

where $\nu_{y}$ is zero current tune. We now assume $\sigma_{x}+$ $\sigma_{y}=2 \bar{\sigma}$ to be independent of $s$, which is approximately true in a typical alternating gradient (AG) focusing structure. We also retain the ripple in the space-charge term due to the betatron function. Thus, the equation of motion can be rewritten as

$$
\ddot{v}+\nu_{y}^{2} v=\frac{\hat{I}}{\sigma_{y} \bar{\sigma}}(1+p \cos n \phi) v,
$$

where $p \cos n \phi$ represents the $\phi$ variation in the lattice, with $n$ being a multipole of the lattice periodicity $\left(4,8,12, \ldots\right.$ in the SNS case), and $\hat{I} \equiv I \bar{\beta}^{2} \nu_{y}^{2}$. The corresponding envelope equation is

$$
\ddot{\sigma}_{y}+\nu_{y}^{2} \sigma_{y}=\frac{\hat{I}}{\bar{\sigma}}(1+p \cos n \phi)+\frac{\epsilon_{y}^{2}}{\sigma_{y}^{3}} .
$$

For the matched beam, we have

$$
\nu_{y}^{2}=\frac{\hat{I}}{\sigma_{y} \bar{\sigma}}+\frac{\epsilon_{y}^{2}}{\sigma_{y}^{4}} .
$$

Taking $\sigma_{y}=\bar{\sigma}(1+z)$, we obtain the equation for small beam oscillations,

$$
\ddot{z}+\left(\nu_{y}^{2}+\frac{3 \epsilon_{y}^{2}}{\bar{\sigma}_{y}^{4}}\right) z=\frac{\hat{I}}{\bar{\sigma}^{2}} p \cos n \phi,
$$

where we have assumed antisymmetric beam envelope oscillation with $x$ and $y$ motion $180^{\circ}$ out of phase. We should note that, for a round beam with the identical horizontal and vertical tunes, there are both symmetric and antisymmetric modes. One would expect that the antisymmetric mode is most likely to get excited due to the AG focusing. When the tunes are sufficiently split, the motion in $x$ and $y$ is essentially decoupled, which modifies the expression for the envelope frequency $\Omega_{2}=2 \bar{\nu}$. This will be taken into account later in our numerical estimates. We now rewrite the above equation as

$$
\ddot{z}+4 \bar{\nu}_{y}^{2} z=\frac{\hat{I}}{\bar{\sigma}^{2}} p \cos n \phi,
$$

where $\bar{\nu}_{y}=\nu_{y}-3 \Delta \nu_{y} / 4$ with $\Omega_{2}=2 \bar{\nu}$ being the envelope frequency. Thus, we have the following solution for $\sigma_{y} / \bar{\sigma}=1+z$ :

$$
\frac{\sigma_{y}}{\bar{\sigma}}=1+\frac{\hat{I}}{\bar{\sigma}^{2}} \frac{p \cos n \phi}{4 \bar{\nu}_{y}^{2}-n^{2}}+C \cos \left(2 \bar{\nu}_{y} \phi+\alpha\right),
$$

where $C$ is some constant of integration. From Eq. (A8), the spectrum of the oscillations of $\left\langle y^{2}\right\rangle$ should contain the following harmonics: $n, 2 n, n+2 \bar{\nu}_{y},\left|n-2 \bar{\nu}_{y}\right|$, $2 \bar{\nu}_{y}, 4 \bar{\nu}_{y}$, etc. We now try to identify these frequencies from the frequency spectra obtained from numerical calculations. Simulations were carried out with a KV beam for the previous FODO SNS lattice with a working point $\left(\nu_{x}, \nu_{y}\right)=(5.82,5.77)$ and a space-charge tune shift of about 0.08 . We note that in the Appendix we analyze the data based on simulations with the ACCSIM code $[1,14]$. A detailed comparison of ACCSIM with ORBIT done by Jeon [7] showed that tunes in the vertical direction are slightly less depressed with the ACCSIM code by approximately $\delta \nu=0.015$ compared to the ORBIT code. As a result, the emittance exchange is still possible in simulations with the ACCSIM code while with ORBIT $\nu_{y}=5.77$ already 
falls outside of resonance bandwidth as shown in Fig. 1. For this reason, an equivalent emittance exchange with the ORBIT code may be obtained by slightly adjusting the vertical zero current tune to $\nu_{y}=5.79$. We now proceed with the analysis of the frequency spectra. The spectrum of $\left\langle y^{2}\right\rangle$ is presented in Fig. 24 in the frequency range from 1 to 25 . First, we identify major peaks at $n=4,8,12,16$, 20 , and 24 which are the harmonics of the lattice with the superperiodicity 4 . The other peaks should come from the superposition of $n$ and $2 \bar{\nu}_{y}$, as described above. Also, the amplitude of the peaks in the frequency spectrum should decrease with the increase of harmonic number $n$. We note that the frequency $\bar{\nu}_{y}$ of the antisymmetric envelope mode occurs for the round beam when tunes in $x$ and $y$ are close to one another. In the numerical example under investigation, $\nu_{x}-\nu_{y}=0.05$ and $\Delta \nu / 4 \approx 0.02$, so that we expect decoupled motion in $x$ and $y$ with the envelope frequency $\Omega_{2}=2 \bar{\nu}_{y}=2\left(\nu_{y}-5 \Delta \nu / 8\right)$. This slightly affects the numerical value of $\bar{\nu}_{y}$ which we need to use but does not change our general discussion about the sidebands of the major harmonics. By assuming $\bar{\nu}_{y} \approx 5.725$, we expect

$$
\begin{aligned}
11.45 & \cong 2 \bar{\nu}_{y}, \\
7.45 & \cong 2 \bar{\nu}_{y}-4, \\
15.45 & \cong 2 \bar{\nu}_{y}+4, \\
3.45 & \cong 2 \bar{\nu}_{y}-8,
\end{aligned}
$$

and small peaks (due to the superposition with large values of $n$ ),

$$
\begin{aligned}
12.55 & \cong 24-2 \bar{\nu}_{y} \\
8.55 & \cong 20-2 \bar{\nu}_{y} \\
4.55 & \cong 16-2 \bar{\nu}_{y} \\
19.45 & \cong 2 \bar{\nu}_{y}+8 \\
23.45 & \cong 2 \bar{\nu}_{y}+12 \\
0.55 & \cong 12-2 \bar{\nu}_{y} \text { (not plotted) } .
\end{aligned}
$$

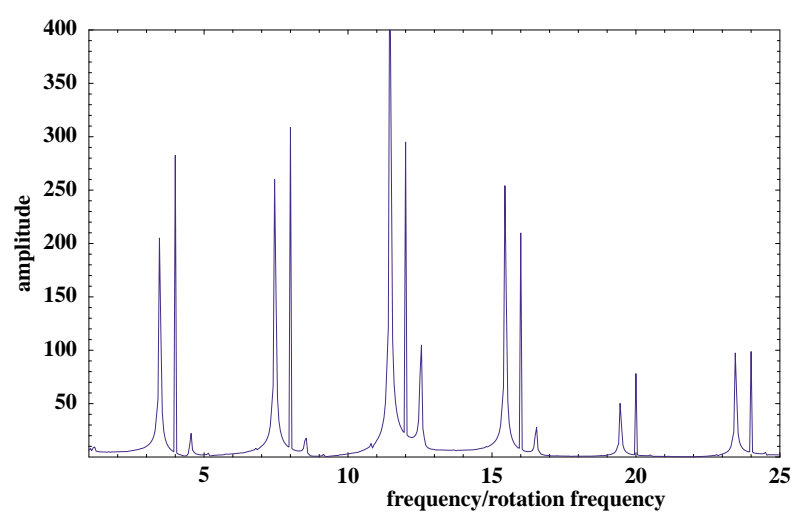

FIG. 24. (Color) Spectrum of $\left\langle y^{2}\right\rangle$ after 250 turns (working point $5.82,5.77)$.
These numbers are the sidebands observed in Fig. 24. Also, there is no visible evidence of $4 \bar{\nu}_{y}=22.9$, which suggests that $C$ in Eq. (A8) is small.

For an ideally populated symmetrical KV distribution we expect $\langle y\rangle$ to be zero, which is confirmed by the $\langle y\rangle$ spectrum. As follows from Eq. (A3), an asymmetric bunch should have harmonics $n \pm \bar{\nu}_{y}$ (no combinations with $2 \bar{\nu}_{y}$ ).

\section{B. Horizontal motion}

To correctly predict the allowed frequencies in beam oscillations, we must include the effect of dispersion. We start with the Hamiltonian without space charge,

$$
H=\frac{p_{x}^{2}}{2}+\frac{K_{x}(s)}{2} x^{2}+\frac{m^{2} c^{4}}{E_{0}^{2}} \delta^{2}-\frac{x}{\rho(s)} \delta,
$$

where the longitudinal momentum is $p_{0}(1+\delta)$. The equation of motion in $x$ is

$$
x^{\prime \prime}+K_{x}(s) x=\frac{\delta}{\rho(s)},
$$

with $\delta \equiv \Delta p / p_{0}$. The solution of this equation is

$$
x(s)=x_{\beta}(s)+D(s) \delta,
$$

where $x_{\beta}$ is a general solution describing betatron motion, and satisfies

$$
x_{\beta}^{\prime \prime}+K_{x}(s) x_{\beta}=0 .
$$

The term $D(s) \delta$ in Eq. (A13) is a particular solution of Eq. (A12) where $D(s)$ refers to the local dispersion function and satisfies

$$
D^{\prime \prime}+K_{x}(s) D=\frac{1}{\rho(s)} .
$$

We now use the transformation $D=\sqrt{\beta_{x}} B$, $d \phi=d s /\left(\beta_{x} \nu_{x}\right), x(s)=\sqrt{\beta_{x}} u(\phi)$ to obtain

$$
\ddot{u}+\nu_{x}^{2} u=0, \quad \ddot{B}+\nu_{x}^{2} B=\frac{\nu_{x}^{2} \beta_{x}^{3 / 2}}{\rho(s)} .
$$

To include space-charge terms we again assume a KV-like beam and obtain

$$
\begin{gathered}
\ddot{u}+\nu_{x}^{2} u=\nu_{x}^{2} \beta_{x}^{2} \frac{2 I}{\sigma_{x}\left(\sigma_{x}+\sigma_{y}\right)} u, \\
\ddot{B}+\nu_{x}^{2} B=\nu_{x}^{2} \beta_{x}^{2} \frac{2 I}{\sigma_{x}\left(\sigma_{x}+\sigma_{y}\right)} B+\frac{\nu_{x}^{2} \beta_{x}^{3 / 2}}{\rho(s)} .
\end{gathered}
$$

Similar to the vertical motion, we thus have

$$
\begin{gathered}
\ddot{u}+\bar{\nu}_{x}^{2} u=\frac{\hat{I} u}{\bar{\sigma}^{2}} p \cos n \phi, \\
\ddot{B}+\bar{\nu}_{x}^{2} B=\frac{\hat{I} B}{\bar{\sigma}^{2}} p \cos n \phi+\nu_{x}^{2} q \cos n \phi,
\end{gathered}
$$

where $q \cos n \phi$ comes from the periodic variation of $1 / \rho$. Thus, for a bunch which starts out with symmetry $\left(\left\langle x_{\beta}\right\rangle=\right.$ 0 ), oscillations of $\langle x\rangle$ should contain natural frequencies of $D: n, n \pm \bar{\nu}_{x}$. This is confirmed by frequency spectrum 


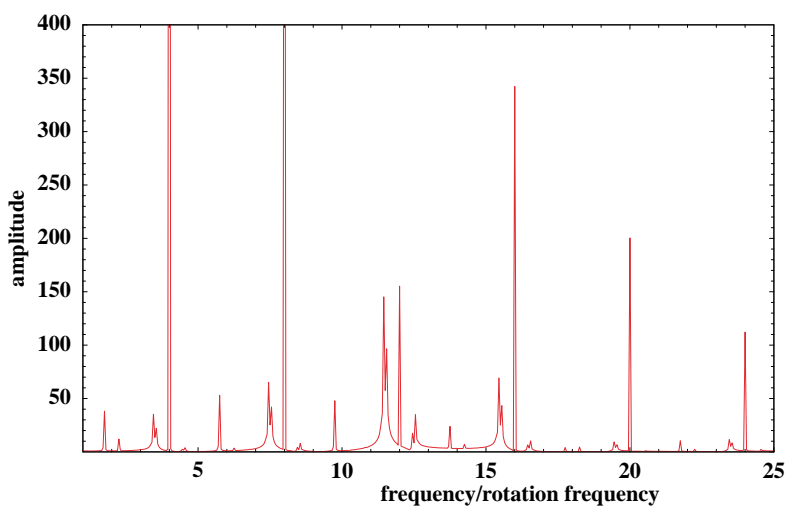

FIG. 25. (Color) Spectrum of $\left\langle x^{2}\right\rangle$ after 250 turns.

of $\langle x\rangle$. For the frequencies of envelope oscillations $\left\langle x^{2}\right\rangle$, we have

$$
\left\langle x^{2}\right\rangle=\left\langle x_{\beta}^{2}\right\rangle+2\left\langle x_{\beta} D\right\rangle \delta+\delta^{2}\left\langle D^{2}\right\rangle .
$$

In addition to harmonics $n, n \pm 2 \bar{\nu}_{x}, 4 \bar{\nu}_{x}$, we now expect peaks at $n \pm \bar{\nu}_{x}$ which should be relatively small because they are proportional to $\delta$. This is again confirmed by Fig. 25. Some discussion of the combined effect of space charge and $\Delta p / p$ on the tune spread of the SNS beam can be found in $[12,13]$.

\section{Application to observed fourth order structure}

The $y$ - $p_{y}$ phase space snapshot taken at 250 turns resembles the structure of a fourth order resonance (similar to the phase-space plots presented in the main body of this paper). Simulations were performed without magnet errors and, therefore, exclude the appearance of arbitrary harmonics in beam oscillations. Hence, due to the superperiodicity 4 of the lattice, $n=23$ is not allowed. Also, the above analysis and the simulation results both show that superposition of lattice harmonics $n$ with $\bar{\nu}_{y}$ does not produce harmonic 23 . Thus, no fourth order nonstructure lattice resonance is expected for the matched beam based on the above analysis.

The analysis developed above was based on the envelope equation, which correctly describes only second order beam modes. In addition, the beam can have higher-order modes. High-order coherent mode frequencies can be calculated using the Vlasov equation. For a KV beam in the smooth focusing approximation they are available in the literature $[2,15]$. The inclusion of AG flutter does not significantly change the eigenfrequencies unless we are near the lattice resonances [16]. Thus we can apply these coherent frequencies to the lattice under discussion. The general form of these frequencies is $\Omega_{m}=m\left(\nu_{0}-C_{m} \Delta \nu\right)$. The coefficient $C_{m}$ is significantly different from unity only for $m=2$, especially for a symmetric mode. In the case of a fourth order antisymmetric mode we have

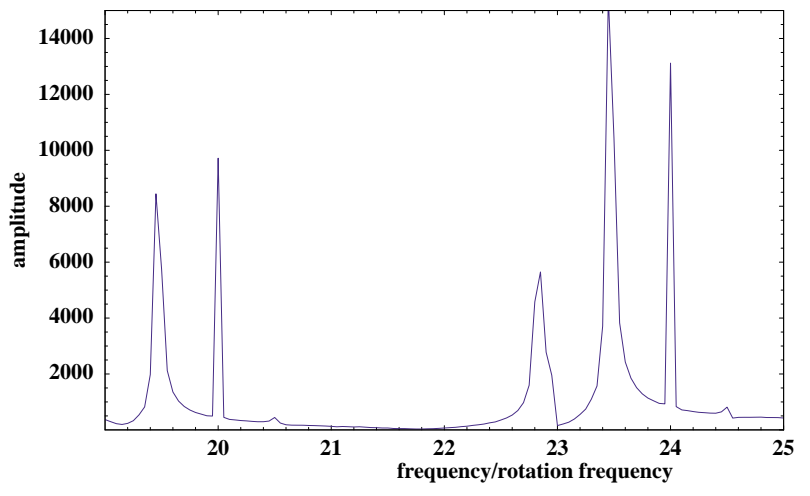

FIG. 26. (Color) Spectrum of $\left\langle y^{4}\right\rangle$ after 250 turns.

$\Omega_{4}=4\left(\nu_{0}-\frac{31}{32} \Delta \nu\right)$. With our previous assumption of $\bar{\nu}_{y} \approx 5.7$ we thus have $\Omega_{4} \approx 22.8$. In fact, Fig. 26 shows a peak around this number. Based on this spectrum analysis, the nature of the observed resonance appears to be associated with the development of a high-order collective beam mode driven by the space-charge coupling resonance rather than with the lattice harmonics. At a later stage of the calculation, we observe the resonance between individual particles with depressed tune and the fourth order coherent beam mode $\left(4 \tilde{\nu}_{y}=\Omega_{4}\right)$ as these particles enter the halo.

[1] D. Jeon, J. A. Holmes, V. V. Danilov, J. D. Galambos, and D. K. Olsen, Phys. Rev. E 60, 7479 (1999).

[2] I. Hofmann, Phys. Rev. E 57, 4713 (1998).

[3] I. Hofmann, J. Qiang, and R. D. Ryne, Phys. Rev. Lett. 86, 2313 (2001).

[4] J. Wei, D. T. Abell, J. Beebe-Wang, M. Blaskiewicz, P. Cameron, N. Catalan-Lasheras, G. Danby, A. V. Fedotov, C. Gardner, J. Jackson, Y. Y. Lee, H. Ludewig, N. Malitsky, W. Meng, Y. Papaphilippou, D. Raparia, N. Tsoupas, W. T. Weng, R. L. Witkover, and S. Y. Zhang, Phys. Rev. ST Accel. Beams 3, 080101 (2000).

[5] J. Galambos, J. Holmes, D. Olsen, A. Luccio, and J. BeebeWang, ORBIT User's Manual, SNS/ORNL/AP Technical Note No. 011, 1999.

[6] I. Hofmann (private communication).

[7] D. Jeon (private communication).

[8] F. W. Jones, in Proceedings of the 1997 Particle Accelerator Conference, Vancouver, Canada (IEEE, Piscataway, NJ, 1997), p. 2597.

[9] B. W. Montague, CERN Report No. 68-38, 1968.

[10] R. L. Gluckstern, W. H. Cheng, and H. Ye, Phys. Rev. Lett. 75, 2835 (1995).

[11] I. Hofmann and O. Boine-Frankenheim (to be published).

[12] Y. Papaphilippou, in Proceedings of the Particle Accelerator Conference, Chicago, 2001 (to be published).

[13] A. V. Fedotov, N. Malitsky, Y. Papaphilippou, J. Wei, and J. Holmes, in Proceedings of the Particle Accelerator Conference, Chicago, 2001 (Ref. [12]). 
[14] J. A. Holmes, V. V. Danilov, J. D. Galambos, D. Jeon, and D. K. Olsen, Phys. Rev. ST Accel. Beams 2, 114202 (1999).

[15] R. L. Gluckstern, in Proceedings of the 1970 Linear Accel- erator Conference, Chicago, edited by M. R. Tracy (Fermilab, Batavia, IL, 1970), p. 811.

[16] I. Hofmann, L. J. Laslett, L. Smith, and I. Harber, Part. Accel. 13, 145 (1983). 